\title{
Chromaticities of Lovibond Glasses
}

\section{By Geraldine Walker Haupt and Florence Lesch Douglas}

\begin{abstract}
This paper reports by means of a series of graphs the chromaticity coordinates of certain Lovibond unit glasses and of pairs of such glasses in combination with an illuminant of color temperature $2,842^{\circ} \mathrm{K}$ (illuminant $A$ ). The chromaticities were computed according to the 1931 International Commission of Illumination (ICI) standard-observer and coordinate system [1]. $\quad$ The points graphically representing the $x$ - and $y$-values have been labelled according to the Lovibond numbers and have been connected by lines to form a network. A similar graph is included for a few Lovibond glasses in combination with illuminant $C$. The Lovibond glasses are those from the National Bureau of Standards set designated as "BS9940", and the spectrophotometric data on which the colorimetric computations are based are those published by Gibson and Harris [2].
\end{abstract}

\section{Purpose}

These graphs were prepared to supply additional colorimetric data about the set of Lovibond glasses, BS9940, in possession of this Bureau and, insofar as these data are representative of all sets of Lovibond glasses, to describe further the Lovibond color system.

Single Lovibond glasses or combinations of glasses may be used to match the chromaticities of a large number of luminous and transparent substances. Although it is important that glasses be available to make such chromaticity matches, it is equally important that the chromaticities so obtained be described in fundamental terms. Graphs of this type make this fundamental description possible, for they afford a rapid method for converting "Lovibond number" to trichromatic coordinates, and vice versa.

\section{Lovibond Glasses}

\section{General Description}

Sets of Lovibond glasses were first made available as color standards sometime after 1896 , when the company, The Tintometer, Ltd., was organized. A complete set of Lovibond glasses consists of a set each of red, yellow, and blue glass slides, which absorb selectively chiefly in the green, violet, and orange regions, respec-

\footnotetext{
${ }^{1}$ Figures in brackets indicate the literature references at the end of this paper.
}

tively, of the visible spectrum. Each set of colored glasses is graded by a series of numbers that are intended to be proportional to the amount of the colorant flashed onto the slides. Thus a combination of any two glasses of the same nominal hue is approximately equivalent to a single glass whose series number is equal to the sum of the series numbers of the two component glasses; for example, two Lovibond glasses numbered 5 red and 3 red are intended to be equivalent in chromaticity to a glass numbered 8 red. In addition to the nominal additivity of the glasses of each of the three hues, the amounts of colorants used in the respective series are such that the combination of a red, a yellow, and a blue glass of the same number gives a chromaticity (with daylight illuminant) that is approximately achromatic.

The glasses normally available in the three sets of colors have nominal values ranging as follows: $0.01,0.02$ to $0.20 ; 0.22,0.24$ to $0.90 ; 0.95,1.00$ to $2.0 ; 2.1,2.2$ to $5.0 ; 5.2,5.3$ to 11.0 ; and 11.5 to 20.0. Each glass slide is approximately $5 \mathrm{~cm}$ long and $1.7 \mathrm{~cm}$ wide; the thicknesses of the glasses vary from 1 to $3 \mathrm{~mm}$. The computations reported in the present paper are based upon spectrophotometric data previously published [2] for the Lovibond unit glasses (1.0, 2.0 to 20.0) together with a more detailed description of the Lovibond system.

Earlier colorimetric studies [3] have shown that red glasses from different sets are frequently 
quite different, even though they are numbered alike. Further studies on the inconsistencies between red glasses from different Lovibond sets [4] resulted in the development of a method for regrading the red glasses so that their new numbers are consistently additive when used in combination with Lovibond 35 yellow [5]. This method was based on colorimetric data derived from the spectrophotometric measurements on the red unit glasses in set BS9940. Furthermore, these spectrophotometric data provided a means of determining the extent to which the series numbers ascribed to the glasses in any one set fail to be proportional to the logarithms of the spectral transmittances. These inconsistencies within and between sets explain why the Optical Society of America Committee on Colorimetry considered it more appropriate to discuss the Lovibond system as a subtractive colorimeter, that is, the tintometer, rather than as a system of color standards [6].

Although the inconsistencies in the grading of the Lovibond glasses are often considerably greater than the least perceptible difference associated with a particular color, the glasses, nevertheless, furnish a practical set of color standards that has become increasingly useful in a wide variety of industrial applications. They may be used to match the chromaticities of a large number of transparent and luminous substances, the comparisons being made between the light transmitted by the Lovibond glasses and the light transmitted or emitted by the substance of unknown chromaticity. Although the range of chromaticities obtainable with Lovibond glasses does not extend to those of highest purity, it, nevertheless, covers a larger area on the International Commission on Illumination (ICI) chromaticity diagram than is covered by any other set of material color standards for which comparable data are available. The use of these glasses not only enables many chromaticity matches to be made, but enables such matches to be made conveniently in rather small hue steps.

In recent years the demand for calibrations of red glasses has decreased sharply, apparently because the need for such standards in the vegetable oil industry has already largely been met. On the other hand, although fewer in number, these recent requests for standardization of Lovibond red glasses represent a diverse group of industries. In the Bureau's work, typical uses of the Lovibond glasses are (1) colorimetric measurements required for obtaining duplicates of the red and yellow lantern glasses for certification as railroad signal standards, (2) determinations of the colors of neon lights and luminous flares, (3) check of the locations of the hue-name boundaries on the ICI diagram according to the ISCC-NBS system [7], and determination of chromaticity limits for the signal color designated as identification lunar-white, which were suggested, and later adopted, as Amendment 1 to the Army-Navy Specification Colors, $\mathrm{AN}-\mathrm{C}-56$.

By means of the graphs described below, we have thus extended our use of the Lovibond glasses to obtain approximate chromaticity coordinates $(x, y)$ of any unknown color within the Lovibond range. The method is to compare the unknown color (of a transparent filter, or of a light source, such as a pyrotechnic, or a phosphorescent or fluorescent material) with various combinations of Lovibond glasses with illuminant $A$ until a satisfactory match is obtained, or until by interpolation, the Lovibond notation of the unknown is found. Then the chromaticity coordinates of the unknown are read from the graph. To the extent that the Lovibond numerals are reliable, these graphs can serve the same purpose for combinations of Lovibond glasses possessed by other investigators.

We have also used these graphs to facilitate our use of combinations of Lovibond glasses as chromaticity standards in our measurements of chromaticity coordinates $(x, y)$ by means of the chromaticity-difference colorimeter [8]. In this application, the graph aids in the choice of the chromaticity standard agreeing most closely with that of the unknown, and the graph also serves as a convenient source of the chromaticity coordinates of the standard chosen.

\section{National Bureau of Standards Set}

In 1913 the Society of Cotton Products Analysts, now American Oil Chemists' Society, agreed that the National Bureau of Standards set of Lovibond glasses, set BS9940, be adopted as the standard and that all their glasses be compared with glasses of this standard set to eliminate the inconsistencies found in the grading of edible oils. Spectral transmittance measurements were accordingly made on the 20 red unit glasses in order to 
supply the fundamental data required for their standardization. While similar measurements were made at the same time on the 20 unit blue and yellow glasses, only the red glasses, needed by the oil industry, were standardized. These red glasses have been used at this Bureau in calibrating more than three thousand [9] of the Lovibond red glasses for use in the grading of vegetable and cottonseed oils alone. ${ }^{2}$

Further importance is attached to this set as the spectrophotometric data on these 60 glasses have been used as the basis for conversion charts published by The Tintometer, Ltd [10]. Their graphs are to be used for converting the tintometer instrumental readings to ICI chromaticity coordinates for certain Lovibond glasses, singly and in paired combinations, with illuminants $B$ and $C$, respectively. According to R. K. Schofield [11], the chromaticities shown on the graphs "were computed from three spectrophotometric curves which are now the ultimate standards to which the glasses approximate very closely," and these curves are defined ${ }^{3}$ by the spectrophotometric data published by this Bureau. ${ }^{4}$

\section{Permanence of Lovibond Glasses}

The spectrophotometric data used in making the computations for the present paper were made in 1923. The spectral transmittances of 13 glasses of set BS9940, including the unit red glasses, 12.0,

\footnotetext{
2 The colors of vegetable oils vary in general from pale yellow to deep reddish orange. The Lovibond red glasses by themselves are purplish red and do not match the oil colors unless combined with Lovibond yellow glasses. Over a considerable range the vegetable oil colors are adequately matched by a combination of Lovibond 35 yellow and variable red, and all of the Lovibond red glasses recalibrated for the vegetable oil industry have been standardized on this basis. A comparison of vegetable oil colors and Lovibond yellow plus red colors is given in a paper by H. J. MeNicholas, Color and spectral transmittance of vegetable oils, J. Research NBS 15, 99 (1935) RP815, and Oil \& Soap 12, 167 (1935).

${ }^{3}$ A letter from G. S. Faw cett, Managing Director, The Tintometer, Ltd., dated November 7, 1944, states that "the data published in BS Sei. Pap. S547 formed the basis for computing the nomographs for standard illuminants $B$ and $C$ which we have published. Irregularities in the curves were smoothed out and the data on certain glasses which show marked irregularities or were noted as being defective by the undersigned when he visited the Bureau in 1931, were entirely ignored in averaging for the true values. These defective glasses are 3.0, 4.0, 7.0, 20.0 red, and 17.0, 20.0 blue. A part from these glasses, which undoubtedly are not up to present-day standards, we feel any other slight irregularities in the curves can well be due to the smallness of the area tested, particularly as they are flashed glasses and very small striae or other defects would have a relatively big effect on such a small area, and that your tests were made about the geometrical center of the glass and our tests only take into account the lower third of the glass."

4 The set BS9940 consists of 470 glasses. This paper contains data for only 60 of them. These are the ones numbered 1.0, 2.0 to 20.0, in the three colors. Extensive measurements have been made on several of the tenths and hundredths glasses of this set, but these data have not been published.
}

13.0, 17.0, and 20.0, were remeasured in 1930 and again in 1938. The maximum difference in the $x$ - or $y$-values of these four-unit glasses for the three sets of measurements is $0.001_{7}$, as shown in table 1. These discrepancies are to be attributed to improved methods of measuring spectral transmittances rather than to any change in chromaticity with time. This is considered further below.

TABLE 1.-Comparisons of chromaticity coordinates and luminous transmittances obtained from three sets of measurements on four Lovibond red glasses in combination with illuminant $A$

\begin{tabular}{|c|c|c|c|c|}
\hline \multirow{2}{*}{ Glass No. } & \multirow{2}{*}{$\begin{array}{c}\text { Date of } \\
\text { measure- } \\
\text { ments }\end{array}$} & \multicolumn{2}{|c|}{$\begin{array}{l}\text { Chromaticity co- } \\
\text { ordinates }\end{array}$} & \multirow{2}{*}{$\begin{array}{l}\text { Lumi- } \\
\text { nous } \\
\text { transmit- } \\
\text { tance }\end{array}$} \\
\hline & & $x$ & $y$ & \\
\hline & 1923 & $0.566_{1}$ & $0.345_{6}$ & 0.363 \\
\hline \multirow[t]{3}{*}{12 red. } & 1930 & $.565_{1}$ & $.345_{9}$ & .363 \\
\hline & 1938 & $.565_{6}$ & $.344_{6}$ & .359 \\
\hline & 1923 & $.571_{3}$ & $.343_{5}$ & .347 \\
\hline \multirow[t]{3}{*}{13 red... } & 1930 & $.570_{4}$ & $.344_{3}$ & .348 \\
\hline & 1938 & $.570_{6}$ & $.343_{2}$ & .345 \\
\hline & 1923 & .5968 & $.336_{9}$ & .270 \\
\hline \multirow{3}{*}{17 red } & 1930 & $.595_{2}$ & $337_{3}$ & .270 \\
\hline & 1938 & $.595_{4}$ & $.336_{6}$ & .267 \\
\hline & 1923 & $.605_{0}$ & .3320 & .257 \\
\hline \multirow{2}{*}{20 red.. } & 1930 & $.604_{6}$ & $.332_{2}$ & .256 \\
\hline & 1938 & $.605_{6}$ & $.330_{5}$ & .250 \\
\hline
\end{tabular}

\section{Effect of Temperature on the Colors of Lovibond Glasses}

The effect of a temperature increase of $25^{\circ} \mathrm{C}$ on the chromaticities of Lovibond glasses $35 \mathrm{Y}, 7.2 \mathrm{R}$, and $35 \mathrm{Y}$ plus $7.2 \mathrm{R}$, has been reported [12] as being approximately the same as adding 0.2 Lovibond red units. No comparable study has been made for the blue glasses but unpublished spectral transmission data ${ }^{5}$ for a cobalt blue glass, approximately equivalent in chromaticity to a Lovibond blue glass of 18 value, show that the $x$ - and $y$-coordinates change by amounts equal to $+0.000_{7}$ and $+0.001_{0}$, respectively, when the temperature of the glass is raised from $25^{\circ}$ to $50^{\circ} \mathrm{C}$. It is probable, therefore, that the temperature changes that Lovibond glasses undergo during normal conditions of usage cause only negligible changes in their chromaticities.

\footnotetext{
- Data obtained by Lois A. Peterson on a cobalt blue glass, $3.00 \mathrm{~mm}$ in thickness, designated as "Corning G55A $\alpha^{19}$ ". This glass is one of the spectrophotometric standards in possession of the National Bureau of Standards.
} 


\section{Discussion of the Graphs}

Figure 1 shows the chromaticity coordinates, according to the 1931 ICI standard observer and coordinate system, of the 20-unit glasses in each of the red, yellow, and blue series, each glass in combination with ICI standard illuminant $A$ (color temperature $2,842^{\circ} \mathrm{K}$ ), and, for the same illuminant, the chromaticity coordinates of various combinations of the unit glasses, each combination consisting of two unit glasses of different colors. This figure orients the Lovibond network with respect to the spectrum and Planckian loci on the ICI diagram and shows the four divisions of the net work which have been drawn to a larger scale in figures 3 to 6 , inclusive. Data similar to those in figure 1 are shown in figure 2, except that ICI standard illuminant $C$ (representative of average daylight) is used instead of illuminant $A$, and fewer points are shown. This graph should be useful for comparisons with the similar graph of smoothed data which has been prepared by R. K. Schofield of The Tintometer, Ltd. Figures 3, 4, 5 , and 6 are sections of figure 1 drawn to a larger scale as warranted by the accuracy of the data. These sections, moreover, show the chromaticities of many more combinations of the Lovibond glasses than are shown in the orientation chart of figure 1 .

The chromaticity loci for the red, yellow and blue series extend radially from the points repre-

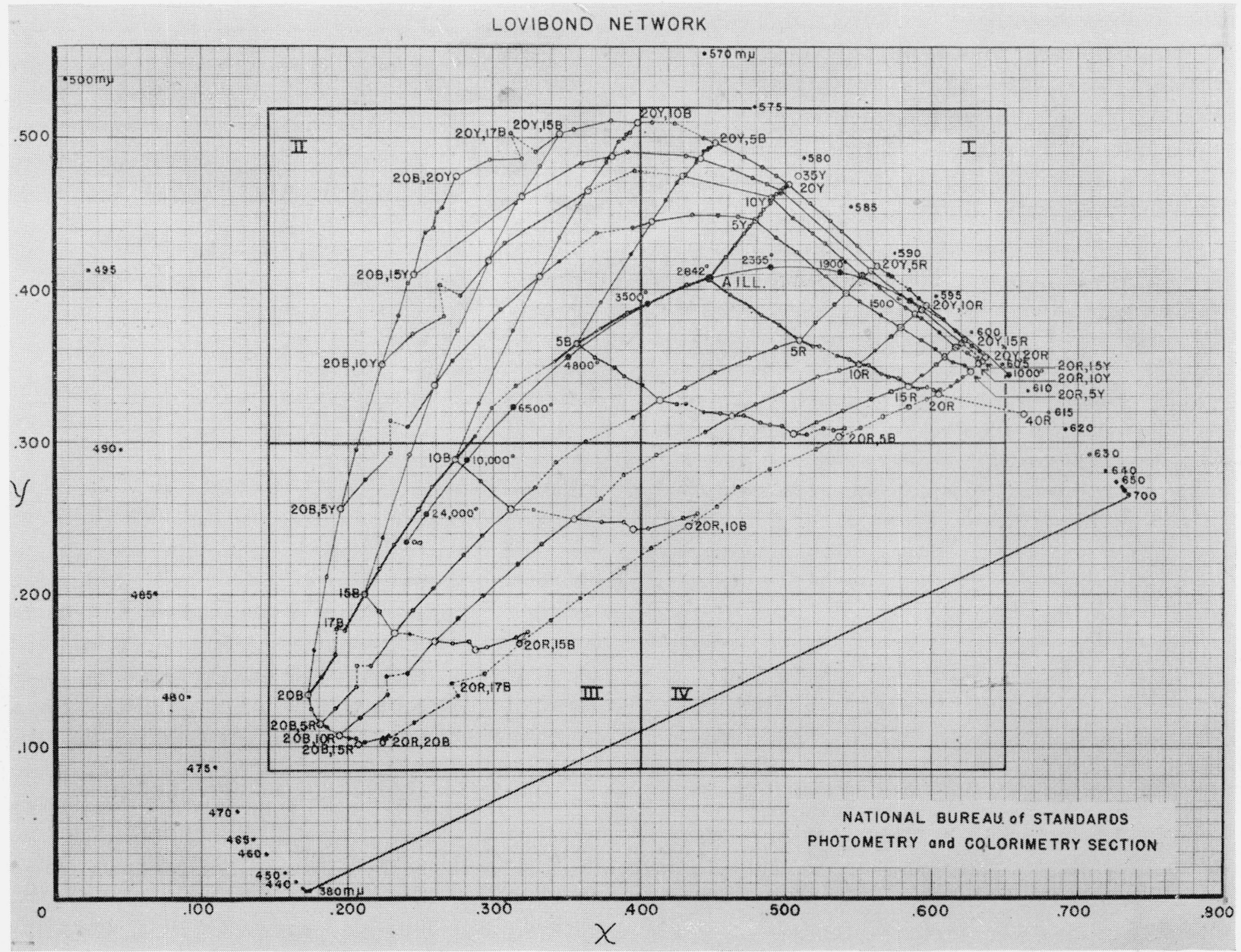

Figure 1.-Chromaticities $(x, y)$, in terms of the 1931 International Commission on Illumination (ICI) standard observer and coordinate system, of Lovibond red, yellow, and blue glasses, singly and in pairs, (yellow, red; blue, yellow; red, blue) as indicated, when combined with ICI illuminant $A\left(2,842^{\circ} \mathrm{K}\right.$, representative of incandescent light).

The four sections of this diagram are reproduced to larger scale in figures $3,4,5$, and 6 , wherein the chromaticities of many additional pairs of glasses are also shown. 


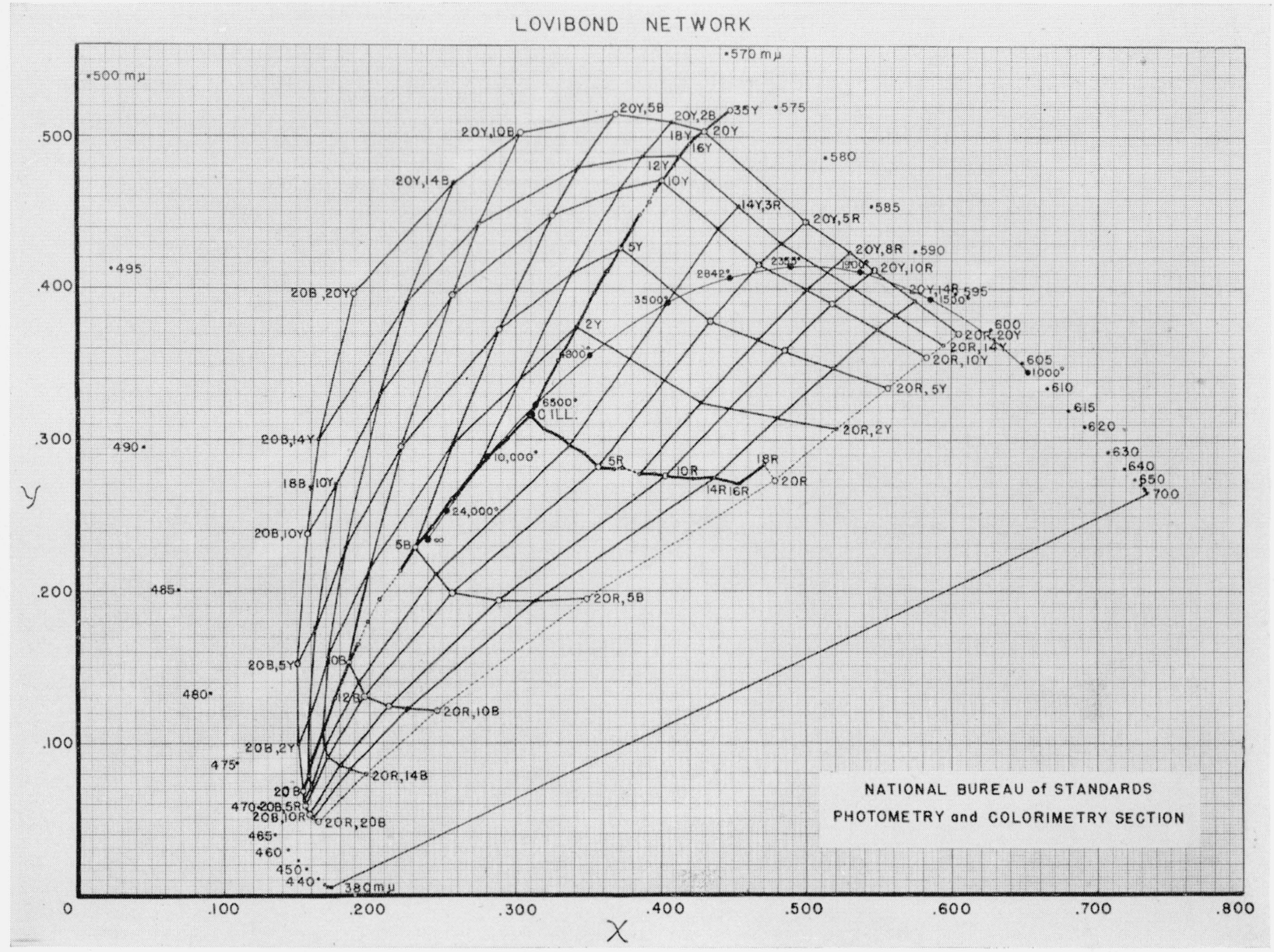

FIGURE 2.- Same as figure 1, except that the illuminant is ICI illuminant $C$ (approximately $6,500^{\circ} \mathrm{K}$, representative of average daylight).

senting illuminants $A$ or $C$ and form boundaries between the areas wherein are plotted the chromaticities of the combinations. The combinations red-yellow, yellow-blue, and blue-red yield chromaticities comprising a network within the yellow-red, green, and purple areas, respectively, on the mixture diagrams [1]. The points representing the chromaticities of the glasses or combinations of glasses have been connected by straight lines since these data represent exactly only the unit Lovibond glasses from set BS9940. It will be seen that such chromaticity loci might be drawn fairly well as smoothed curves, thereby masking most of the irregularities found in the actual glasses. Smoothed curves, based on data from particular glasses of á particular set, however, do not satisfy the additivity condition [5] exactly, and, on this account, are less useful than are curves based on an "ideal" Lovibond system in which the chromaticities of the glasses and their combinations are consistent with the Lovibond numbering.

In figure 1, it will be noted that along the chromaticity locus for the Lovibond reds, the glasses numbered 7, 17, and 20 seem certainly irregular; similarly, 7 and 8 blue are slightly inconsistent, 17 blue is strikingly irregular, and 8 yellow is probably the most irregular yellow glass.

These deviations from the regular trend of the chromaticities apply only to these particular glasses of the BS9940 set; they are not to be thought of as characteristic of like numbered Lovibond glasses in general. Consequently, the straight lines leading from any of these deviate points are dashed. Other lines considered to be 


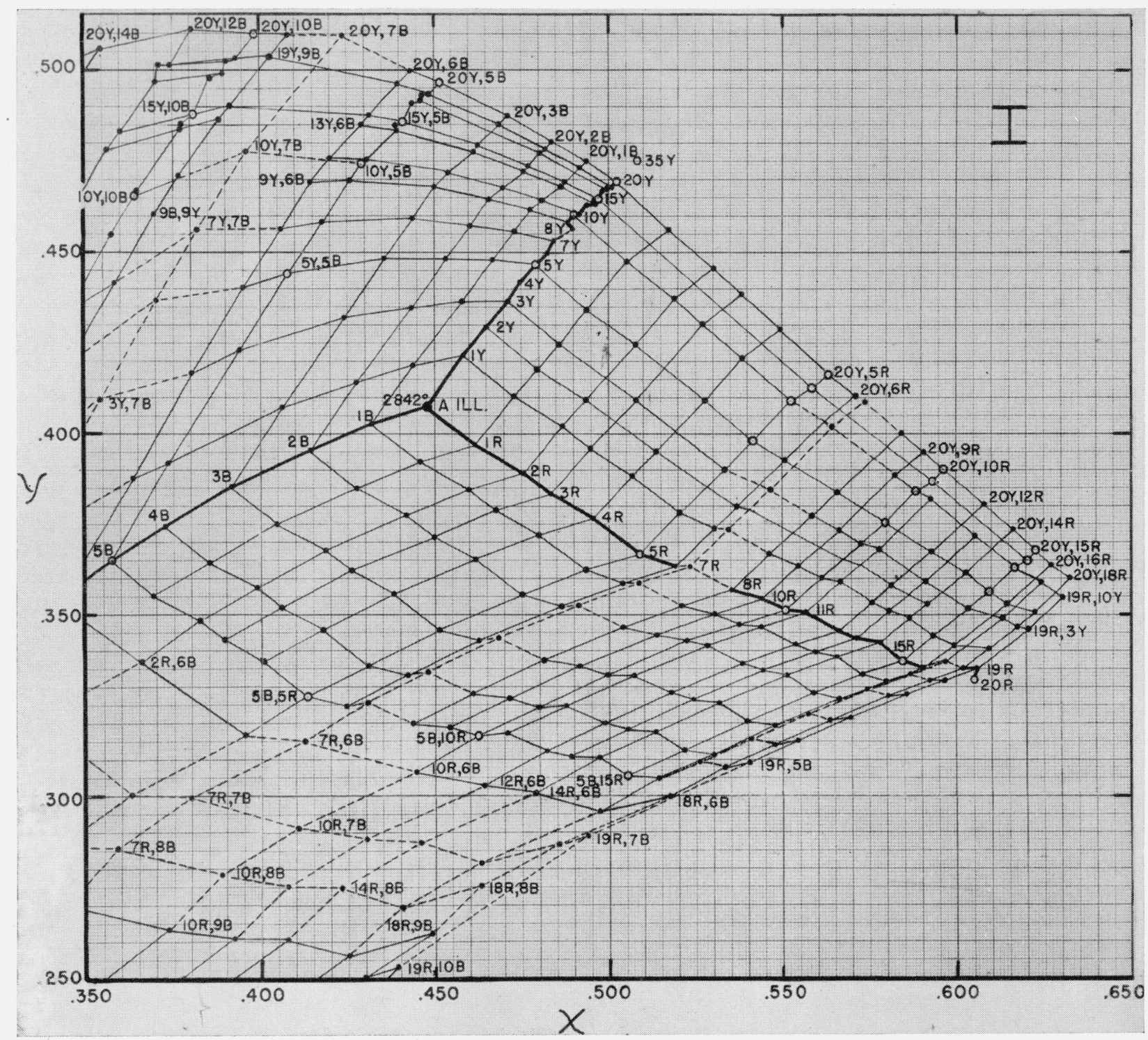

FIGURE 3.-Enlarged graph of section I of figure 1.

a fairly safe guide to the chromaticities characteristic of the Lovibond system as a whole are indicated by solid lines. ${ }^{6}$

Furthermore, lines based on 17 blue and 20 red are omitted from the enlarged sectional drawings of figures $3,4,5$, and 6 as they are obviously the

\footnotetext{
${ }^{6}$ The conversion charts published by The Tintometer, Ltd. contain curves in which the irregularities have been smoothed out and the data for certain notably irregular glasses have not been used. Computations are in progress at this Bureau for determining the spectral transmittances of ideal Lovibond No. 1.00 glasses for each of the red, yellow and blue series. These idealized spectral transmittances are derived by weighted averages from the measured spectral transmittances of the 20-unit glasses in each of the 3 colors, and will provide the basis for computing "ideal" Lovibond networks typical of all the Lovibond glasses manufactured with set BS9940 as the standard.
}

least "ideal" of the glasses, and their inclusion would, at best, complicate interpolations being made from the graphs, and, at worst, introduce errors that may not be typical of all similarly numbered glasses. Dashed lines are used in the large-scale drawings to join all points involving the four glasses, 7 and 17 red, and 7 and 8 blue, as a reminder that the straight lines, joining glasses consecutively numbered to these irregular ones, cannot be used in interpolating for intermediate points with the same degree of accuracy as elsewhere on the charts. 


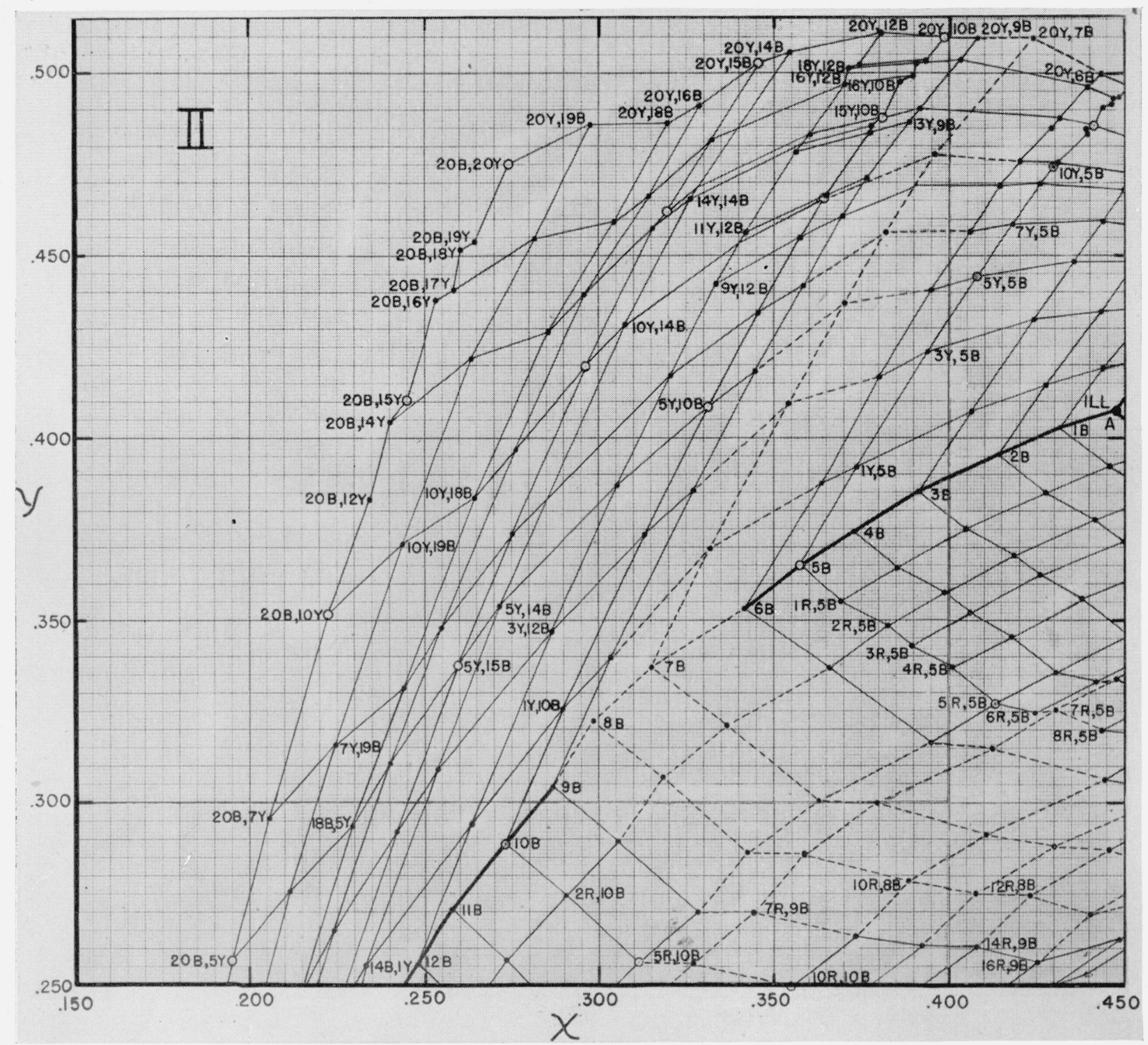

FiguRE 4.-Enlarged graph of section II of figure 1.

The irregularities in the graphs led to a reconsideration of the spectrophotometric measurements that had been made for certain red glasses and to additional measurements for certain blue glasses. The chromaticities of the red and blue glasses, to a greater extent than those of the yellow glasses, might be affected by impermanence of the glasses or by improved technics of measurement.

The original spectrophotometric measurements were made in 1923 on the Koenig-Martens spectrophotometer without using rotating sectors in those wavelength regions where the transmittances were very low. ${ }^{7}$ In 1930 and again in 1938, the transmittances of many of the glasses of set BS9940 were remeasured on the Koenig-Martens equipment with the 10-percent and 1-percent sector disks at the low transmittances. These remeasured glasses include four of the red unit glasses,

\footnotetext{
${ }^{7}$ For very low transmittances the analysing nicol must be turned close to the extinction points for photometric match. In these positions, stray unpolarized light in the field becomes relatively important and the nicol has to be turned significantly farther towards the extinction points than would be true if the unpolarized light were not present. This results in erroneously low values of transmittance being obtained. Accuracy is restored by the use of rapidly rotating sectors of known aperture in the blank beam, the angle of photometric match being thus returned to a part of the scale known to give reliable results.
} 


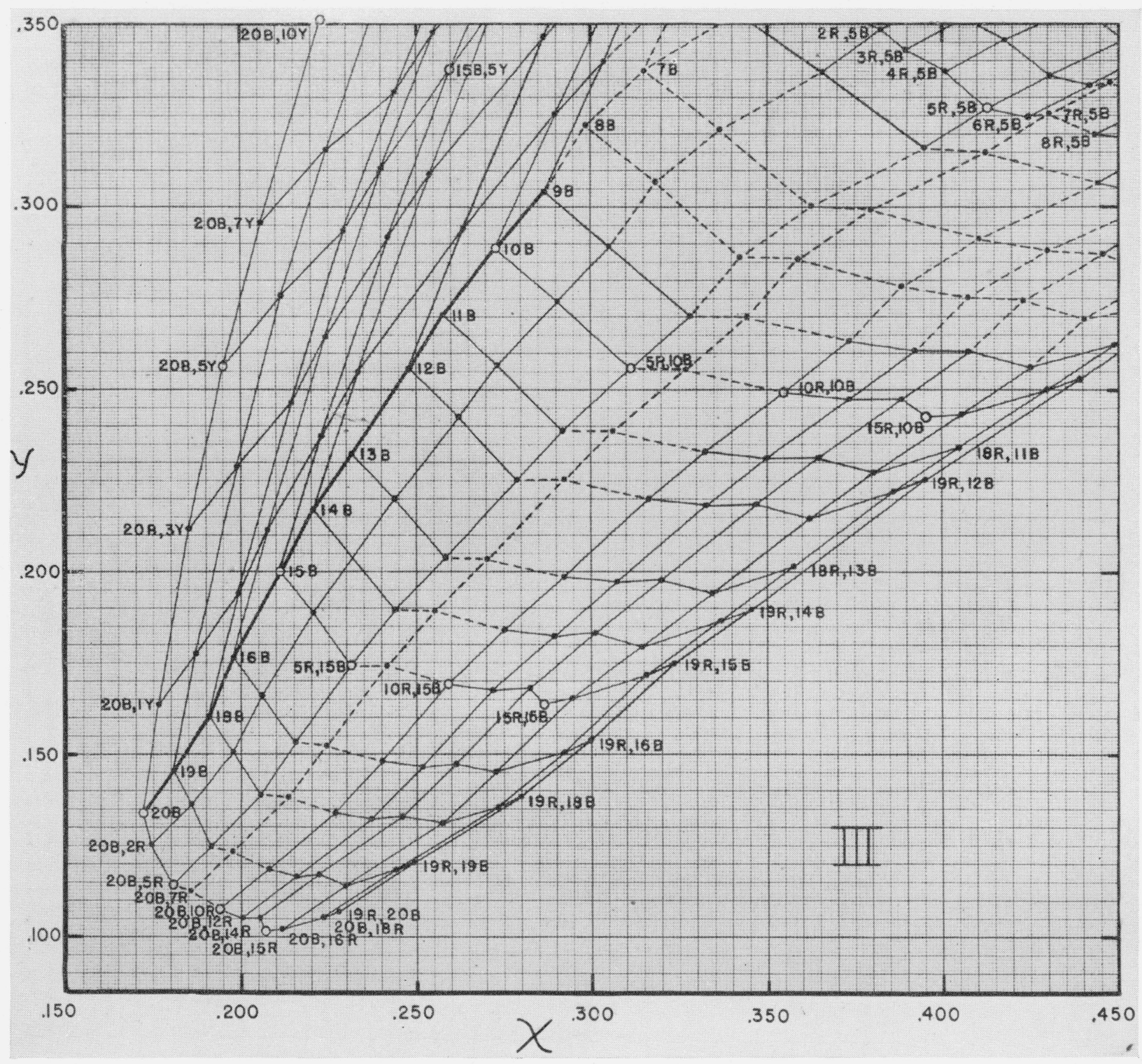

Figure 5.-Enlarged graph of section III of figure 1.

$12.0,13.0,17.0$, and 20.0 ; values for their chromaticity coordinates and luminous transmittances are summarized in table 1 . With the improved technic in 1930, higher spectral-transmittance values were obtained in the region from 500 to $550 \mathrm{~m} \mu$, and these higher values tended to yield chromaticities of slightly lower purity. The 1938 measurements were further improved by the elimination of certain possible errors in wavelength calibrations. The maximum variation in $x$ and $y$ values in all the sets of measurements was only $0.001_{7}$, however, and this is not considered of enough importance, for the purposes of the present paper, to substitute later measurements for the 1923 data.

Spectral transmittances of the 18 and 20 blue glasses were remeasured in 1945 by using the rotating sectors at all wavelengths where the values reported in S547, and measured in 1923, were less than 10 percent. These new measurements were substituted for the corresponding measurements made in 1923, and the computations for the $x$ - and $y$-values were revised accordingly. The results of these computations are shown in table 2 . 


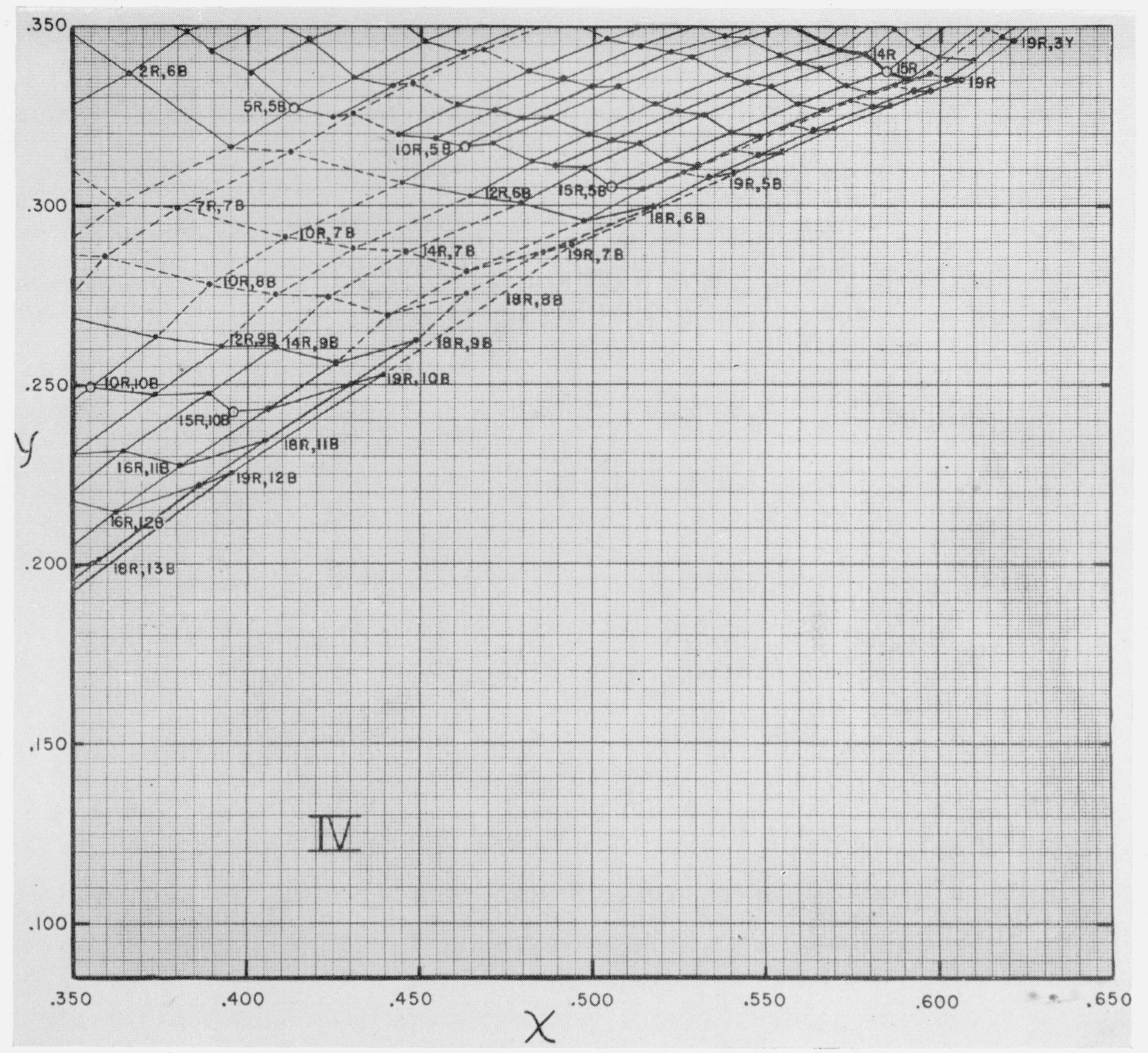

FIGURE 6.-Enlarged graph of section IV of figure 1.

TABLE 2.-Comparisons of values obtained for two Lovibond blue glasses, in combination with illuminant $A$, from measurements made with and without rotating sectors

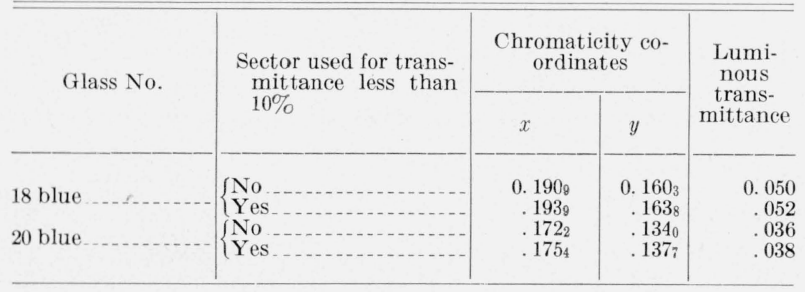

\section{Summary}

This paper presents a series of graphs showing the chromaticities, computed according to the 1931 ICI standard observer and coordinate system, of certain Lovibond unit glasses from set "BS9940" and of pairs of such glasses. There are five graphs (one general, four large-scale sections) for illuminant $A$ (representative of gas-filled incandescent lamps), and one general graph for illuminant $C$ (representative of average daylight). 


\section{References}

[1] Deane B. Judd, J. Opt. Soc. Am. 23, 359 (1933).

[2] K. S. Gibson and F. K. Harris, BS Sci. Pap. 22, 1 (1927) S547.

[3] Irwin G. Priest, Report of Proceedings of the Fourth Annual Meeting of the Society of Cotton Products Analysts, p. 6 to 14 (June 1913); The Cotton Oil Press 4, 43 (1921).

[4] Deane B. Judd and Geraldine K. Walker, Oil \& Fat Industries 5, 16 (1928); and Geraldine K. Walker, BS J. Research 12, 269 (1934) RP653.

[5] Kasson S. Gibson and Geraldine Walker Haupt, BS J. Research 13, 433 (1934) RP718.
[6] Committee on Colorimetry of the Optical Society of America, J. Opt. Soc. Am. 35, 1 (1945).

[7] Kenneth L. Kelly, J. Opt. Soc. Am. 33, 627 (1943).

[8] Deane B. Judd, Textile Research 9, 253 (1939).

[9] Geraldine Walker Haupt, Oil \& Soap 15, 282 (1938).

[10] R. K. Schofield, J. Sci. Inst. 16, 74 (1939); Colorimetry: Part 1, Lovibond System, Part 2, LovibondSchofield System (The Tintometer, Ltd., Milford, Salisbury, Eng.).

[11] G. S. Fawcett, Proc. Phys. Soc. LVI, 8 (1944). (See also p. 20 of "Discussion.")

[12] Deane B. Judd, BS J. Research 1, 859 (1928) RP31.

Washington, April 24, 1947. 